\title{
Erratum
}

\section{Selfconsistent Diabatic Approach to Dissipative Collective Nuclear Motion}

\author{
K. Niita ${ }^{1}$, W. Nörenberg ${ }^{2}$, and S.J. Wang ${ }^{3}$ \\ 1 Institut für Theoretische Physik, Justus-Liebig-Universität Giessen, \\ D-6300 Giessen, Federal Republic of Germany \\ ${ }^{2}$ Gesellschaft für Schwerionenforschung (GSI), Planckstrasse 1, \\ D-6100 Darmstadt 11, Federal Republic of Germany \\ ${ }^{3}$ Institute of Modern Physics, Academia Sinica, Lanzhou, China
}

Received September 10, 1987

Z. Phys. A - Atomic Nuclei 326, 69-77 (1987)

In the Eqs. (4.5) to (4.7) the necessary references to the statistical equilibrium with energy $\langle H\rangle_{\text {equ }}^{0}$ (excluding the collective kinetic energy) and occupation probabilities $n_{\alpha}^{\text {equ }}(\mathbf{q}, t)$ for fixed collective variables is missing. In order to obtain the correct form of (4.5) and (4.6) one has to replace the term $\sum_{\alpha} \varepsilon_{\alpha}^{(s)} \rho_{\alpha \alpha}$ by

$\langle H\rangle_{\mathrm{equ}}^{0}+\sum_{\alpha} \varepsilon_{\alpha}^{(s)}(\mathbf{q})\left\{\rho_{\alpha \alpha}(t)-n_{\alpha}^{\mathrm{equ}}(\mathbf{q}, t)\right\}$

where terms quadratic in $\rho_{\alpha \alpha}-n_{\alpha}^{\text {equ }}$ are neglected. Thus the variation with respect to $q_{n}$ (at constant entropy $S$ ) yields instead of (4.7)

$$
\begin{aligned}
& \frac{\mathrm{d}}{\mathrm{d} t} \sum_{m} B_{n m} \dot{q}_{m}-\frac{1}{2} \sum_{m m^{\prime}} \frac{\partial B_{m m^{\prime}}}{\partial q_{n}} \dot{q}_{m} \dot{q}_{m^{\prime}} \\
& =F_{n}^{\text {equ }}-\sum_{\alpha} \frac{\partial \varepsilon_{\alpha}^{(s)}}{\partial q_{n}}\left\{n_{\alpha}(t)-n_{\alpha}^{\text {equ }}(\mathbf{q}, t)\right\}
\end{aligned}
$$

where the equilibrium force

$$
F_{n}^{\text {equ }}=-\left(\frac{\partial}{\partial q_{n}}\langle H\rangle_{\text {equ }}^{0}\right)_{S}
$$

at temperatures larger than $2 \mathrm{MeV}$ may be approximated by the liquid-drop energy. Note that any $\dot{\mathbf{q}}-$ dependence of the two-body matrix elements has been ignored. 